\title{
LONG-RANGE-TRANSPORTED MINERAL DUST FROM AFRICA AND MIDDLE EAST TO EAST ASIA OBSERVED WITH THE ASIAN DUST AND AEROSOL LIDAR OBSERVATION NETWORK (AD-NET)
}

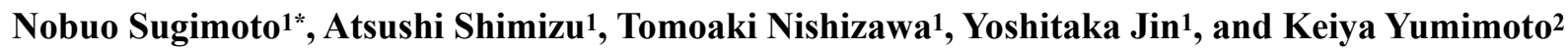 \\ ${ }^{1}$ National Institute for Environmental Studies, 16-2 Onogawa, Tsukuba, Ibaraki 305-8506 Japan \\ ${ }_{2}^{2}$ Research Institute for Applied Mechanics, Kyushu University, Kasuga, Fukuoka, 816-8580 Japan \\ *Email:nsugimot@nies.go.jp
}

\begin{abstract}
Mineral dust generated in Africa and Middle East is sometimes transported to East Asia. Some cases were observed with the Asian Dust and aerosol lidar observation Network (AD-Net). In the large Sahara dust event in March 2018, which was reported by mass media as that snow in Sochi, Russia was stained into orange, the dust was transported to Sapporo, Japan in 4 days from Sochi and observed with the AD-Net lidar. Sahara dust events were also observed in April 2017 and April 2018 with AD-Net. The source areas and transport paths were studied with chemical transport models and trajectory analysis and also confirmed with CALIPSO data. This study showed that long-range transport from Africa and Middle East to East Asia is not rare in springtime.
\end{abstract}

\section{INTRODUCTION}

Continuous lidar network observations have been conducted since 2001 in AD-Net. Currently, lidars are continuously operated at 20 locations in East Asia (Japan, Korea, China, Mongolia and Thailand). The major targets of the observations are Asian dust and regional air pollution aerosols in the lower atmosphere [1].

The source areas of major dust phenomena in East Asia are the Gobi desert in Mongolia and Chinese Inner Mongolia, and the Taklamakan desert in China. Dust from the Gobi is transported near the surface and significantly affect the environment of the lower atmosphere $[2,3]$. It is often mixed with air pollution $[4,5]$. Dust from Taklamakan is often uplifted and transported long distance in the free troposphere [6]

We reported a Sahara dust case observed with the AD-Net lidars in 2005 [7]. We observed lofted dust layer in Suwon and Gosan in Korea and Tsukuba in Japan which was not reproduced by a regional dust transport model. We compared with global aerosol transport models and analyzed with back trajectory analysis, and we found the dust was transported from Africa. We finally confirmed the source region by retrospective simulation separately masking Sahara and Taklimakan. The extinction coefficient estimated at the dust plumes in Gosan and Tsukuba was $\sim 0.05 \mathrm{~km}^{-1}$ at the maximum. After that, we did not pay much attention to long-range-transported dust cases, because our major targets were heavy dust events and regional air pollution.

In 2015-2016, Leibniz Institute for Tropospheric Research (TROPOS) conducted continuous observation with a multi-wavelength Raman lidar in Dushanbe, Tajikistan in Central Asia [8], and they recently started long-term observation. That motivated us to study long-range-transported dust cases again using recent AD-Net data. It would be interesting if we could observe the same air mass along the transport path to study the change in optical characteristics of dust. Also, recent studies of bioaerosols (microbes attached to dust particles) suggest such long-range transport might be important even if the density of the transported dust is low.

\section{METHODOLOGY}

We used the AD-Net two-wavelength (1064 nm and $532 \mathrm{~nm})$ and polarization sensitive $(532 \mathrm{~nm})$ lidar data (http://www-lidar.nies.go.jp/AD-Net/). We firstly used archived results of NAAPS (Navy Aerosol Analysis and Prediction System) of the U.S. Naval Research Laboratory (https:// www.nrlmry.navy.mil/aerosol/) to find possible long-range-transported dust cases to East Asia. We then searched corresponding plumes in ADNet data. When we found corresponding dust plumes, we performed backward trajectory analysis using HYSPLIT (Hybrid Single-Particle Lagrangian Integrated Trajectory model) of U.S. National Oceanic and Atmospheric 
Administration (https://ready.arl.noaa.gov/ HYSPLIT.php). We also confirmed dust transport using the CALIPSO browse data when available. (https://www-calipso.larc.nasa.gov)

\section{RESULTS}

\subsection{Sahara dust case in March 2018}

It was reported by mass medias (BBC, $\mathrm{CNN}$, etc.) that dust from Sahara stained snow in Sochi, Russia into orange on March 22, 2018. We observed the transported dust plume by the lidar in Sapporo on March 26 as shown in Fig.1.
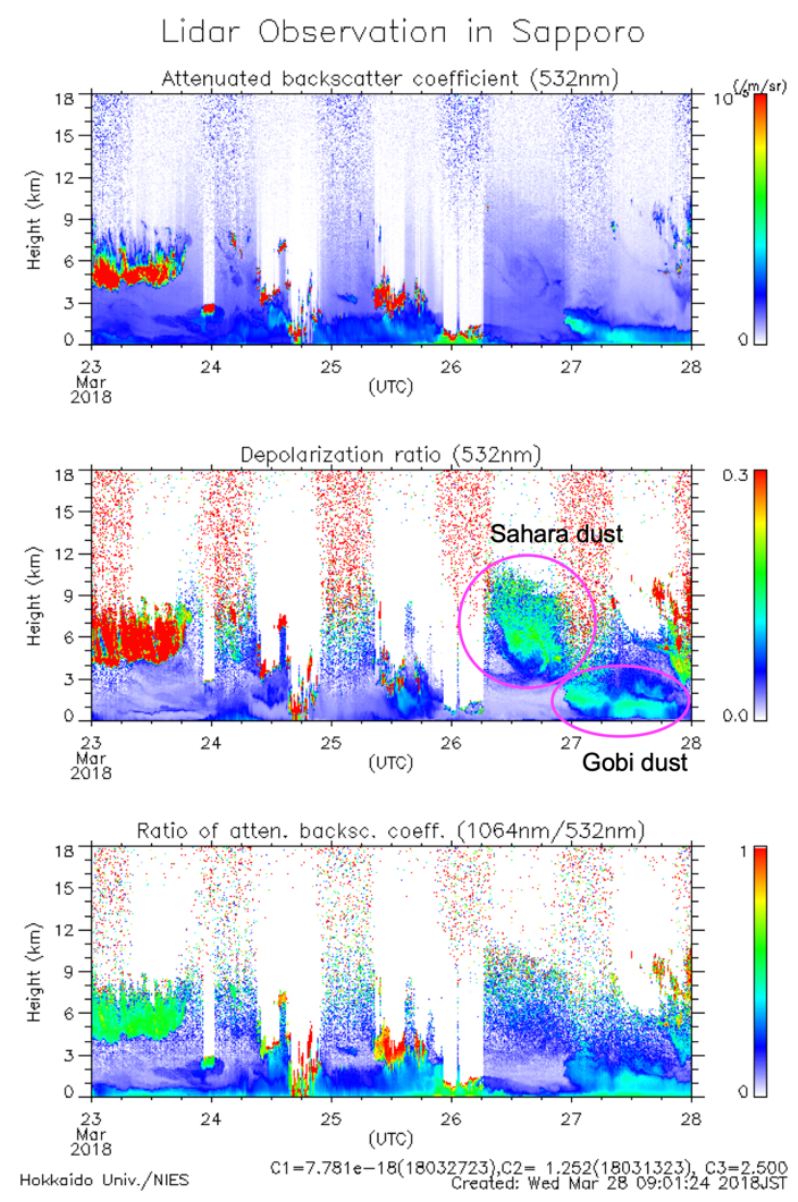

Fig.1 Time-height indications of attenuated backscatter coefficient at $532 \mathrm{~nm}$ (top), volume depolarization ratio at $532 \mathrm{~nm}$ (middle), and color ratio of attenuated backscatter coefficients at $1064 \mathrm{~nm}$ to $532 \mathrm{~nm}$ (bottom), observed in Sapporo, Japan in March 2018.

Figure 2 show the HYSPLIT backward trajectory of the plume. It shows the trajectory exactly passed over Sochi on March 22. The plume was transported through Kazakhstan and Russia.

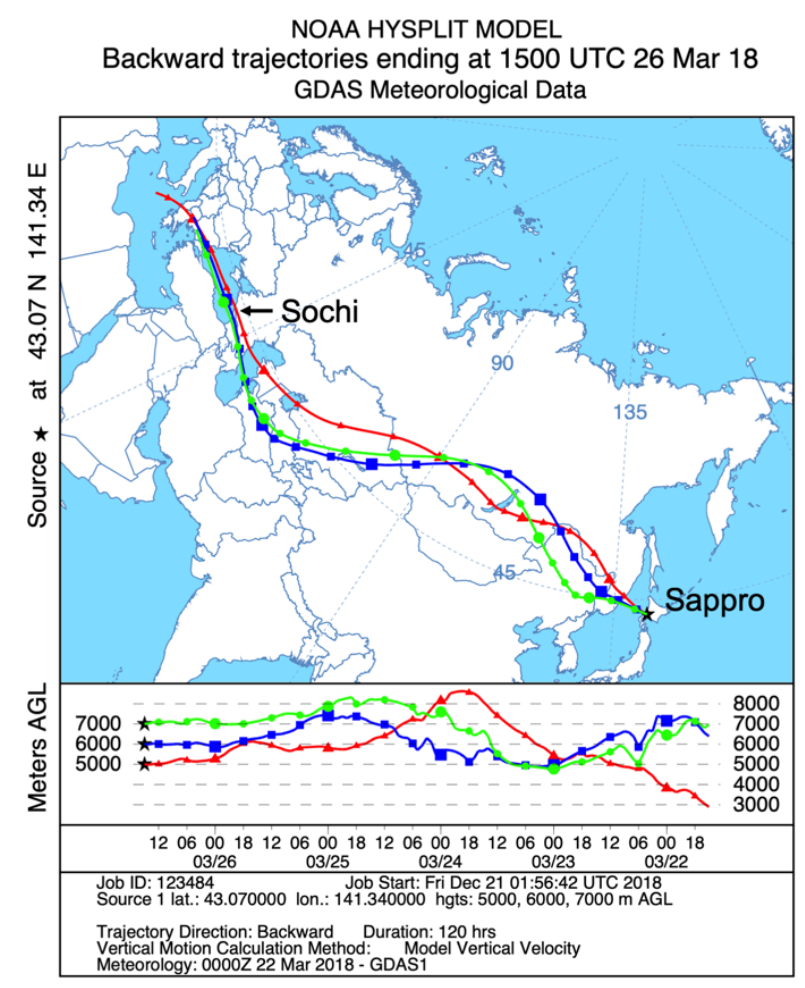

Fig. 2 HYSPLIT backward trajectory of the Sahara dust plume indicated in Fig. 1.

The extinction coefficient of the plume derived with the Fernald method with an assumption of $\mathrm{S} 1=50 \mathrm{sr}$ was $\sim 0.06 \mathrm{~km}^{-1}$ at the maximum. The particle depolarization ratio was $\sim 0.28$. Optical depth of the plume estimated from the lidar extinction coefficient was $\sim 0.3$ at the maximum. Backscattering color ratio $\left(\beta_{1064 \mathrm{~nm}} / \beta_{532 \mathrm{~nm}}\right)$ of the plume was about 0.4 . It is very low compared to that in strong Gobi dust cases $(\sim 1.0)$. This suggests the particle size of the long-rangetransported Sahara dust was small, or the plume was mixed with other aerosols such as sulfate. The result of NAAPS showed however the mixing was not likely. Though it is not possible to estimate the mass concentration quantitatively because the size distribution is not known, in a very rough estimation it was several tens microgram per cubic meter. It was high as a longrange-transported dust. Also, the dust layer stayed above Sapporo for a half day.

\subsection{Sahara dust case in April 2017}

Figure 3 shows another example of Sahara dust observed in Sainshand, Mongolia. A lofted dust plume is seen on April 27-28, 2017, and it looks a part of the dust plume was taken into the 
boundary layer and reached the ground. Estimated extinction coefficient of the dust plume was $\sim 0.05$ $\mathrm{km}^{-1}$, and particle depolarization ratio was $\sim 0.30$. Backward trajectory of the dust plume is shown in Fig.4. Judging from the NAAPS archived results, the emission source of the dust was in Africa. Dust was transported through Kazakhstan and Russia and entered into Mongolia from the northwest. The transport pass was similar to that of March 2005 case [7].
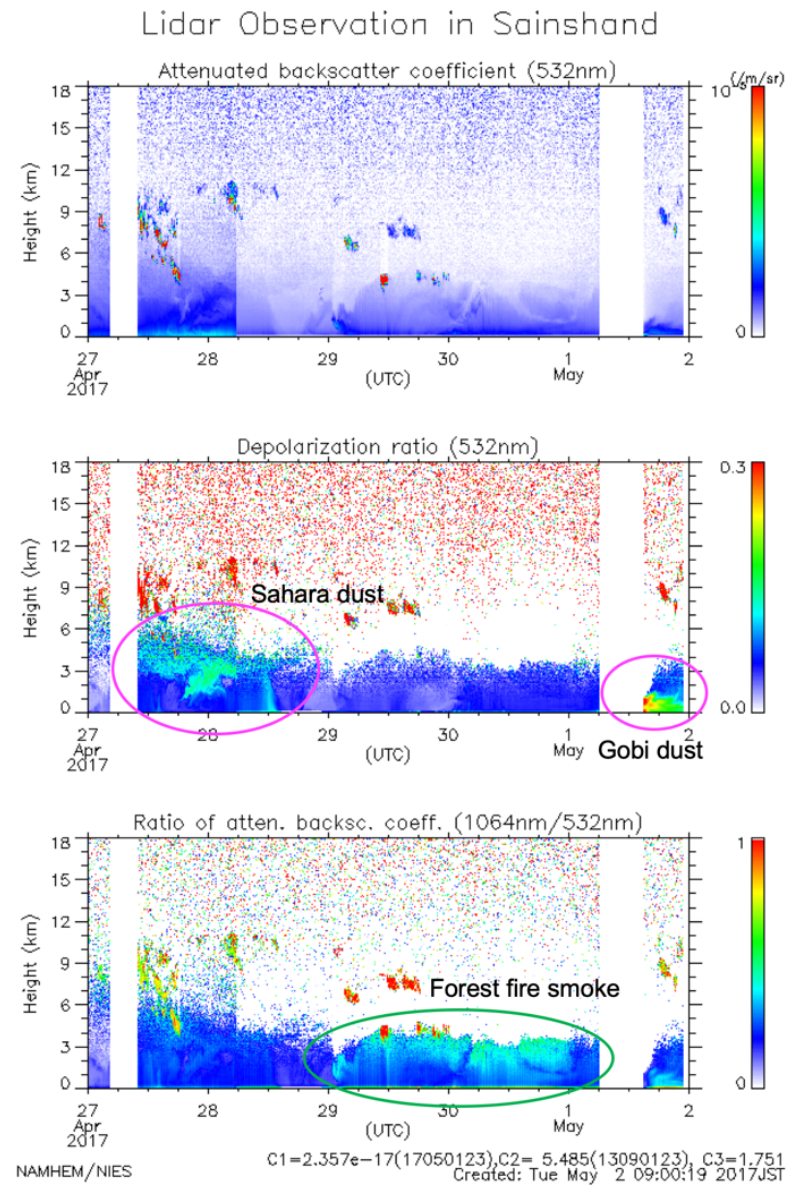

Fig.3 Time-height indications of attenuated backscatter coefficient at $532 \mathrm{~nm}$ (top), volume depolarization ratio at $532 \mathrm{~nm}$ (middle), and color ratio of attenuated backscatter coefficients at $1064 \mathrm{~nm}$ to $532 \mathrm{~nm}$ (bottom), observed in Sainshand, Mongolia in April 2017.

An interesting structure is seen in the color ratio of attenuated backscattering coefficient on April 29 and 30 in Fig.3. The NAAPS results indicate there was forest fire smoke in the boundary layer. The color ratio increased with height because the attenuation at $532 \mathrm{~nm}$ was larger than at $1064 \mathrm{~nm}$.

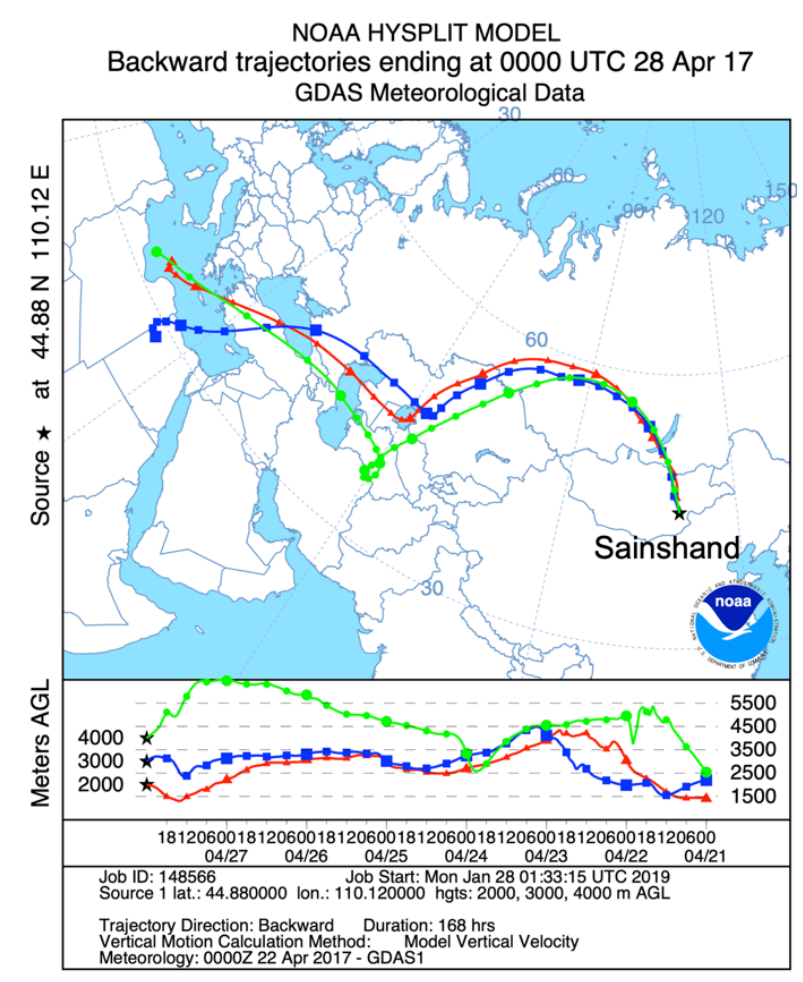

Fig.4 HYSPLIT backward trajectory of the Sahara dust plume indicated in Fig. 3.

\subsection{Middle East dust cases}

We also identified several cases of long-range transport of dust from Middle East. A good example is the case reported by Hofer et al. [8]. They observed a lofted dust layer over Dushanbe, Tajikistan on April 12, 2015. We observed probably the same dust plume in Nagasaki on April 17.

Figure 5 shows backward trajectory arriving at Nagasaki on April 17. The trajectory shows the plume passed near Dushanbe on April 12. The dust was transported across the high mountain in the west of the Taklamakan desert and across the Taklamakan desert.

Another example of Middle East dust was the plume observed in Sendai, Japan on April 27, 2015. In this case the dust was transported through the north side of Mongolia similar to the transport path in Fig.2. 


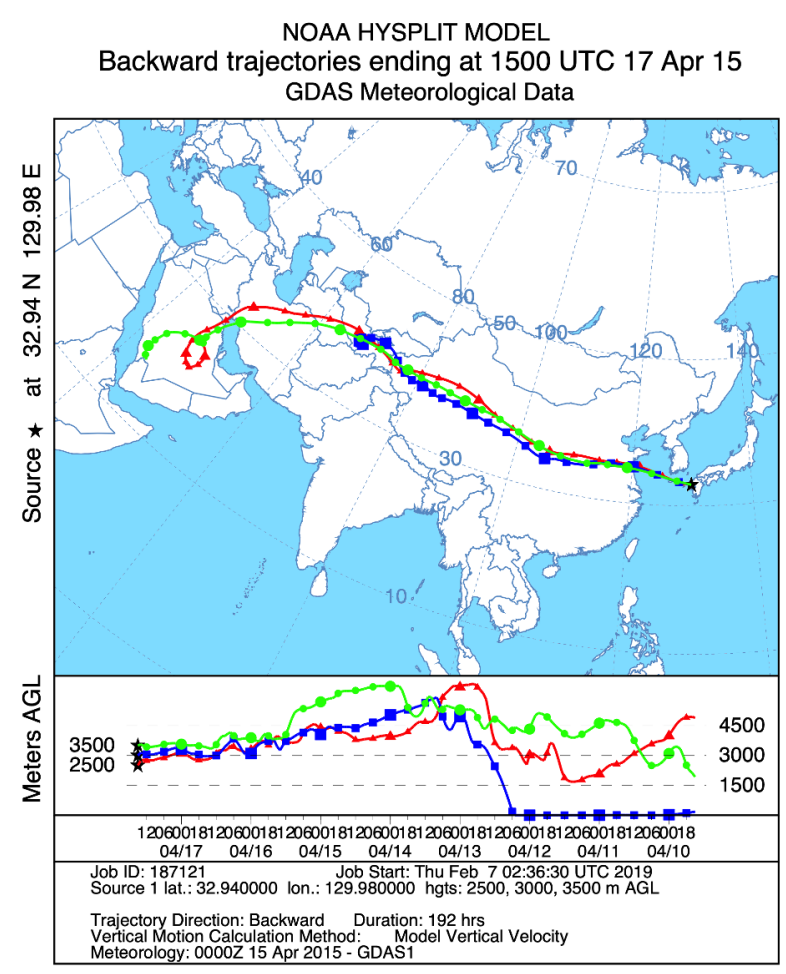

Fig.5 HYSPLIT backward trajectory of the dust observed in Nagasaki on April 17, 2015.

\section{Discussion}

Dust from Africa and Middle East are transported to East Asia every year in the springtime (mostly in March and April). The transport path arriving in East Asia often passes through Kazakhstan and Russia. Dust plumes transported across the high mountain in the west of the Taklamakan desert were also observed. In such cases, however, longrange-transported dust is mixed with the dust from the Taklamakan and the Gobi.

To understand dust emission source and mixing of dust from different sources during transport, we require a global dust transport model having source identification tags. ${ }^{* *}$

In seasons other than spring, no significant longrange-transported dust cases from Africa and Middle East to East Asia have been observed, so far, after 2015. The heavy Central Asia dust events observed in Dushanbe in summer reported by Hofer et al. [8] were not transported to East Asia. Interestingly, those events themselves were not well reproduced in the global aerosol transport models (NAAPS and SPRINTARS).

We realized through this work that Mongolia is a good location also for studying characteristics of long-range-transported dust from Africa and Middle East. We think it is reasonable to improve one of the AD-Net lidars in Mongolia to a multiwavelength Raman lidar or HSRL. Also, it will be very useful if we have an observation site in Kazakhstan.

**Comparison with the global chemical transport model MASINGAR separately calculated for different dust source regions was recently published in Ref.(9).

\section{ACKNOWLEDGEMENTS}

We would like to thank Teppei J. Yasunari, Dashdondog Batdorj, and all other people involved in AD-Net for their contributions in the lidar observations.

\section{REFERENCES}

[1] A. Shimizu, T. Nishizawa, Y. Jin, S.-W. Kim, Z. Wang, D. Batdorj, N. Sugimoto, Opt. Eng. 56 (3), 031219 (2017)

[2] N. Sugimoto, I. Uno, M. Nishikawa, A. Shimizu, I. Matsui, X. Dong, Y. Chen, H. Quan, Geophys. Res. Lett. 30 (12), 1640 (2003)

[3] A. Shimizu, N. Sugimoto, I. Matsui, K. Arao, I. Uno, T. Murayama, N. Kagawa, K. Aoki, A. Uchiyama, A. Yamazaki, J. Geophys. Res. 109, D19S17 (2004)

[4] N. Sugimoto, T. Nishizawa, A. Shimizu, I. Matsui, H. Kobayashi, J. Quant. Spectrosc. Radat. Trans. 150, 107 (2015)

[5] X. Pan, I. Uno, Z. Wang, T. Nishizawa, N. Sugimoto, S. Yamamoto, H. Kobayashi, Y. Sun, P. Fu, X. Tang, Z. Wang, Sci. Rep. 7, 335 (2017)

[6] I. Uno, I., K. Eguchi, K. Yumimoto, T. Takemura, A. Shimizu, M. Uematsu, Z. Liu, Z. Wang, Y. Hara, N. Sugimoto, Nature Geosci. 2, 557 (2009)

[7] C.-B. Park, N. Sugimoto, I. Matsui, A. Shimizu, B. Tatarov, A. Kamei, C.-H. Lee, I. Uno, T. Takemura, D. L. Westphal, SOLA 1, 121 (2005)

[8] J. Hofer, D. Althausen, S. Abdullaev, et al., Atmos. Chem. Phys., 1714559 (2017)

[9] N. Sugimoto, Y. Jin, A. Shimizu, T. Nishizawa, K. Yumimoto, SOLA 15, 257 (2019) 